\title{
Editorial
}

\section{La economía conductual, un campo multidisciplinar}

http://doi.org/10.14718/revfinanzpolitecon.v12.n1.2020.3402

Joan Miguel Tejedor-Estupiñán*

Las aproximaciones científicas desde puntos de vista multidisciplinares y heterodoxos han derivado en replantear los paradigmas impuestos por las perspectivas teóricas más ortodoxas, que en el último siglo han sido puestas a prueba por la evidencia empírica, permitiendo la construcción de nuevas teorías y métodos en las ciencias económicas.

Adam Smith (1776), el padre de la economía política, desarrolló sus teorías económicas basándose en el orden natural de las cosas y las libertades humanas (Ceña Cervantes, 1976). En ese entonces, la economía surge como un resultado del pensamiento filosófico, del derecho y de las matemáticas, y se reconoce como ciencia al poder expresar supuestos y teorías por medio de métodos matemáticos y estadísticos. Desde una aproximación histórica, encontramos la contabilidad como herramienta de los primeros mercaderes y empresarios; la matemática económica, implementada al análisis microeconómico y macroeconómico; la econometría, que combina la teoría económica, la matemática económica y la estadística; y las más recientes teorías inspiradas en la física e incluso la psicología, como es el caso de la econofísica y la economía del comportamiento (Behavioral Economics).

Ashraf, Camerer y Loewenstein (2005) documentan que Adam Smith estableció planteamientos relacionados con la economía del comportamiento, entre los cuales encontramos conceptos como aversión a la pérdida, elección y autocontrol intertemporal, exceso de confianza, altruismo, equidad e interacciones de mercado. En resumen, para Adam Smith, los sujetos económicos no eran agentes racionales desapasionados, puramente interesados, sino seres humanos multidimensionales y realistas.

Los planteamientos desarrollados por Richard H. Thaler le hicieron acreedor del premio Nobel de Ciencias Económicas en el 2017, por su contribución en el campo de la economía conductual, quince años después de que Daniel Kahneman recibiera el mismo galardón por sus contribuciones en esta particular disciplina. Desde el punto de vista

Ph.D. (C) en Economía, Magíster en Derechos Humanos y Economista. Editor de la Revista Finanzas y Política Económica de la Universidad Católica de Colombia. Dirección de correspondencia: Facultad de Economía, Universidad Católica de Colombia, Carrera 13 n. ${ }^{\circ}$ 47-49 (Bogotá, Colombia). Correo electrónico: jmtejedor@ucatolica.edu.co. (D) Orcid: https://orcid.org/0000-0002-2346-3222 
teórico, Thaler (2016) subraya que el problema central subyace en que se tiene una teoría para lograr dos objetivos diferentes, a saber: caracterizar el comportamiento óptimo y predecir el comportamiento real. Desde la perspectiva del trabajo empírico, la disciplina ofrece la oportunidad de estructurar mejores modelos de comportamiento económico, incorporando los supuestos de otras disciplinas y ciencias sociales.

En su artículo "Behavioral Economics: Past, Present, and Future", Thaler (2016) destaca a varios economistas que a inicios del siglo XX desarrollaron las teorías de Adam Smith sobre descuento del tiempo y la teoría económica moderna de elección intertemporal, entre ellos Arthur Cecil Pigou e Irving Fisher. Recuerda también a John Mynard Keynes, que se anticipaba al concepto de comportamiento financiero, al igual que a Vilfredo Pareto y John Maurice Clark, que consideraban que la psicología tenía un rol importante en la economía. A mediados del siglo XX, Herbert Simon (1955) describía que la teoría económica tradicional postula un "hombre económico" que es, a la vez, sujeto "económico" y "racional". Su modelo supone que este hombre conoce las características principales de su entorno, tiene un sistema de preferencias organizado y estable y una habilidad en la computación que le permite calcular según los cursos de acción alternativos que están disponibles para él, los cuales le ayudarán a alcanzar el punto más alto posible en su escala de preferencia (es decir, un escenario donde la maximización de utilidad es sustituida por la satisfacción).

Thaler (2016) describe que la teoría económica neoclásica se basa en los siguientes supuestos específicos sobre el comportamiento humano: a) los agentes tienen preferencias bien definidas, creencias y expectativas imparciales; b) sus decisiones se basan en dichas creencias y preferencias, apoyados en sus capacidades cognitivas infinitas y una fuerza de voluntad infinita; ellos eligen lo que es mejor, mas no lo que es momentáneamente tentador; c) aunque pueden actuar de manera altruista, especialmente hacia amigos cercanos y familiares, su principal motivación es el interés propio. Para el economista, estos son los supuestos que definen el Homo economicus, también llamados Econs. La economía del comportamiento simplemente reemplaza al Econs por el Homo sapiens, también conocido como humano.

Con fundamento en estos referentes, Thaler construye su teoría inspirado también en los trabajos de Amos Tversky y el premio Nobel de Economía en el 2002 Daniel Kahneman (1974), dos psicólogos israelíes de la Universidad Hebrea de Jerusalén, que realizan tres aportes centrales a la disciplina de la economía conductual. En primer lugar, en su artículo "Judgment under Uncertainty: Heuristics and Biases" se enfocan en el juicio humano y en cómo las personas realizan pronósticos y toman decisiones bajo incertidumbre. Los psicólogos usan el término juicios, que los economistas a menudo denominan estimaciones o pronósticos; también heurística, una palabra elegante para las reglas de juego; finalmente subrayan el sesgo (bias), por ejemplo en la información, que hace que las personas cometan errores predecibles (Thaler, 2018).

El segundo aporte conceptual proporcionado por Kahneman y Tversky (1979) es su "teoría de la prospectiva" (Prospect Theory). Mientras la primera investigación se centró en 
los juicios, la segunda se enfocó en las decisiones, particularmente en aquellas que ocurren en entornos de incertidumbre. Esta, de hecho, es una de las más importantes contribuciones a la economía del comportamiento en las últimas cuatro décadas. Según Thaler (2018), este nuevo aporte, además de ofrecer una teoría simple para explicar múltiples anomalías empíricas, podía ilustrar empíricamente dos tipos de teorías completamente diferentes: la normativa y la descriptiva. Por un lado, la teoría normativa hace referencia a una teoría de lo que se considera una elección racional (en lugar de una declaración sobre moralidad). Por otro, la teoría descriptiva solo predice lo que la gente hará dependiendo de las circunstancias. El defecto evidente de la teoría económica neoclásica es que utiliza una teoría para ambas tareas: la teoría de optimización.

La teoría de la prospectiva plantea dos momentos principales de observación: el de edición y el de evaluación en el proceso de selección. En el primero se hace un análisis preliminar de las alternativas, siguiendo un esquema de orden heurístico en el que, al identificar los resultados idénticos, las personas fijan un punto de referencia, y donde los resultados más bajos son considerados como pérdidas y los más altos, ganancias. En el segundo momento, con base en los resultados potenciales y sus respectivas probabilidades, las personas tienen la capacidad de valorar el beneficio y escogen la alternativa que les provea mayor utilidad. La teoría evidencia que las decisiones de las personas están más motivadas a asumir riesgos en ambientes de pérdidas que en ambientes de ganancias (Kahneman y Tversky, 1979).

El tercer aporte de Tversky y Kahneman (1981) tiene que ver con los "efectos marco" (Framing Effects) y sus implicaciones para los modelos del agente racional. Por ejemplo, enmarcar los resultados en términos de riqueza o bienestar general en lugar de en términos de ganancias y pérdidas específicas puede atenuar la respuesta emocional a una pérdida ocasional. Del mismo modo, la experiencia de un cambio negativo puede variar si el cambio se enmarca como una pérdida no compensada o como un costo incurrido para lograr algún beneficio. Desde este punto de vista, los resultados también pueden reflejar la aceptación o el rechazo de la responsabilidad por consecuencias particulares, contexto en el que la delimitación del marco se usa principalmente como instrumento de autocontrol. Cuando el marco influye en la experiencia de las consecuencias, la adopción de un marco de decisión se considera como un acto éticamente significativo.

Thaler (2016) subraya que, desde el origen de las ciencias económicas, los economistas han implementado diferentes herramientas matemáticas y estadísticas; el acceso progresivo a conjuntos de datos cada vez más grandes y ricos ha dado lugar a nuevos métodos que abarcan multiplicidad de experimentos, enfocados sobre todo en avances empíricos más que teóricos. La economía del comportamiento, entonces, es una disciplina basada en la evidencia y enfocada en el desarrollo de investigaciones empíricas. En este sentido, no es algo revolucionario, sino más bien un retorno al tipo de disciplina de mente abierta e intuitiva que fue propuesta inicialmente por Adam Smith, aunque apoyada ahora en herramientas estadísticas y conjuntos de datos cada vez más poderosos. Que desaparezca 
la disciplina de la economía conductual depende de la capacidad de los economistas para centrar su atención en el estudio de los humanos más que en el de los Econs. Finalmente, destaca que la economía del comportamiento y sus aplicaciones tal vez no cambiarán el mundo, pero le darán a la economía una dimensión más humana, permitiendo la creación de teorías que se apliquen tanto a humanos como a Econs, haciendo que esta disciplina sea más sólida, útil y precisa (Thaler, 2018).

Para el caso de Iberoamérica, el II Congreso Internacional de Economía, Banca y Finanzas: "Aplicación de la economía experimental y las finanzas conductuales en las políticas públicas y privadas", organizado por la Universidad de Lima, la Universidad Católica de Colombia y la Universidad Javeriana de Cali, reunió en Lima, Perú, en noviembre del 2019, a investigadores de distintos países de Iberoamérica para tratar sobre economía conductual y sobre finanzas conductuales o del comportamiento ${ }^{1}$. Como resultado de este congreso, se creó la Asociación Iberoamericana de Economía y Finanzas Conductuales (AIEFIC), que estableció una red de cooperación científica con la Revista Finanzas y Política Económica y, en consecuencia, aportó la selección de las cuatro mejores investigaciones presentadas en el evento académico. Estos trabajos constituyen la primera parte de la presente edición de la Revista y, junto a los demás cinco artículos, comprenden investigaciones que aportan más evidencia al campo de la economía conductual y reflejan la nueva tendencia de investigaciones multidisciplinares y empíricas en los diferentes campos de las ciencias económicas y, para nuestro caso, en el campo de las finanzas y las políticas económicas.

\section{REFERENCIAS}

1. Ashraf, N., Camerer, C. F. y Loewenstein, G. (2005). Adam Smith, behavioral economist. Journal of Economic Perspectives, 19(3), 131-145. https://doi.org/10.1257/089533005774357897

2. Ceña Cervantes, J. L. (1976). El orden natural smithrano yla planificación. Problemas del Desarrollo, 7(28), 119-133. https://doi.org/10.22201/iiec.20078951e.1976.28.41891

3. Kahneman, D. y Tversky, A. (1979). Prospect Theory: An analysis of decision under risk. Econometrica, 47 (2), 263-291. http://www.jstor.org/stable/1914185

4. Simon, H. A. (1955). A behavioral model of rational choice. Quarterly Journal of Economics, 69, 99-118. https://doi.org/10.2307/1884852

5. Thaler, R. H. (2016). Behavioral economics: Past, present, and future. Revista de Economía Institucional, 106(7), 1577-1600. https://doi.org/10.1257/aer.106.7.1577

6. Thaler, R. H. (2018). From cashews to nudges: The evolution of behavioral economics. American Economic Review, 108(6), 1265-1287. https://doi.org/10.1257/aer.108.6.1265

1 http://www.ulima.edu.pe/cebyf/agenda/ii-congreso-internacional-ebf?fbclid=IwAR2mHkCHV4TmmEGvs3 JUPneVUCKliGHONKYm5XWqtv2mtOUM7TnbxitCMps 
Editorial

7. Tversky, A. y Kahneman, D. (1974). The inference of intentions from moves in the Prisoner's Dilemma game. Science, 185(4157), 1124-1131. https://doi.org/10.1126/science.185.4157.1124

8. Tversky, A. y Kahneman, D. (1981). The framing of decisons and the psychology of choice. Science, 211 (4481), 453-458. https://doi.org/10.1126/science.7455683 